\title{
Suppression of STIM1 inhibits the migration and invasion of human prostate cancer cells and is associated with PI3K/Akt signaling inactivation
}

\author{
YIBIN ZHOU ${ }^{1}$, PENG GU $^{2}$, JIAN LI ${ }^{3}$, FENG LI $^{4}$, JIN ZHU $^{1}$, PENG GAO $^{1}$, YACHEN ZANG ${ }^{1}$, \\ YONGCHANG WANG ${ }^{1}$, YUXI SHAN ${ }^{1}$ and DONGRONG YANG ${ }^{1}$ \\ ${ }^{1}$ Department of Urology, The Second Affiliated Hospital of Soochow University, Suzhou, Jiangsu 215000; \\ ${ }^{2}$ Department of Urology, Xishan People's Hospital of Wuxi, Wuxi, Jiangsu 214000; \\ ${ }^{3}$ Department of Urology, The First Affiliated Hospital of Bengbu Medical College, Bengbu, Anhui 233000; \\ ${ }^{4}$ Department of Urology, The First Affiliated Hospital of Xi'an Jiaotong University, Xi'an, Shanxi 710061, P.R. China
}

Received March 12, 2017; Accepted July 18, 2017

DOI: $10.3892 /$ or.2017.5961

\begin{abstract}
Store-operated calcium entry (SOCE) plays an important role in the invasion and migration of cancer cells. Stromal-interacting molecule 1 (STIM1) is a critical component in the SOCE. STIM1 has been attracting more and more attention due to its oncogenic potential. STIM1 inhibition suppresses cell proliferation, migration and invasion in a variety of cancer models both in vitro and in vivo. However, the role of STIM1 in prostate carcinogenesis, in particular, in tumor migration and invasion is unclear. Herein, we downregulated STIM1 in prostate cancer cells by lentivirus-mediated short hairpin (shRNA), and then studied its impacts on cell migration and invasion. We found that migration and invasion of prostate cancer cells were significantly inhibited after the suppression of STIM1. Furthermore, we demonstrated that the PI3K/Akt signaling pathway was inactivated by STIM1 knockdown. The PI3K inhibitor LY294002 synergized with STIM1 knockdown to inhibit cell motility. Our results revealed that STIM1 may act as a novel regulator to promote migration and invasion of prostate cancer cells and is associated with the activation of the PI3K/Akt signaling pathway.
\end{abstract}

\section{Introduction}

Prostate cancer ( $\mathrm{PCa}$ ) is the most commonly diagnosed malignancy and the second leading cause of cancer-related deaths in the western male population, while metastasis contributes to

Correspondence to: Dr Yuxi Shan or Dr Dongrong Yang, Department of Urology, The Second Affiliated Hospital of Soochow University, 1055 Sanxiang Road, Suzhou, Jiangsu 215000, P.R. China

E-mail: shyx_1002@163.com

E-mail: doc_ydr@163.com

Key words: prostate cancer, STIM1, migration, invasion, PI3K/Akt majority of deaths $(1,2)$. The five-year survival rate of patients who present with localized disease is $\sim 90 \%$, which was significantly higher than those with metastatic disease. Only 33\% of men presented with metastatic tumors live beyond 5 years. Although prostate cancer treatment has achieved numerous breakthroughs in the last decade, effective therapies to prevent prostate cancer metastasis to other parts of body are still lacking (3). Therefore, novel approaches are urgently needed for the prevention and treatment of the metastasis of prostate carcinoma.

Calcium $\left(\mathrm{Ca}^{2+}\right)$, an intracellular messenger, is indispensable for various cellular functions, such as proliferation, apoptosis and metastasis $(4,5)$. In non-excitable cells, store-operated calcium entry (SOCE) is the major influx of $\mathrm{Ca}^{2+}$ and is regulated by a store-operated calcium channel (SOC), which consists of two important components, stromal-interacting molecule 1 (STIM1) and calcium release-activated calcium channel protein 1 (ORAI1). STIM1 is located in the endoplasmic reticulum (ER) and acts as $\mathrm{Ca}^{2+}$ sensor. Once the $\mathrm{Ca}^{2+}$ store in ER is depleted, STIM1 is activated and forms an oligomer with ORAI1, a transmembrane protein, to form pores for $\mathrm{Ca}^{2+}$ influx (6).

STIM1 participates in a variety of cellular functions, including muscle contraction, the release of neurotransmitters and hormones, gene transcription, cell proliferation and metastasis (7). Recently, STIM1 has attracted more and more attention due to its oncogenic potential. STIM1 inhibition suppresses cell proliferation, migration and invasion in a variety of cancer models both in vitro and in vivo (8-11). However, to date, its function on metastasis of prostate cancer is unclear.

The phosphatidylinositol 3-kinase/protein kinase-B (PI3K/Akt) signaling pathway plays a pivotal role in cell growth, differentiation, proliferation and metastasis. PI3K phosphorylates and activates Akt, affecting its downstream signaling molecules. It has been demonstrated that activation of Akt promotes migration of tumor cells (12-14). Numerous studies have revealed that the PI3K/Akt signaling pathway is extensively activated in migration and invasion of 
various types of cancers, including liver, breast and pancreatic cancer (15-17). PI3K/Akt inhibition by specific inhibitors suppresses cell migration and invasion (18-21). Whether the $\mathrm{PI} 3 \mathrm{~K} / \mathrm{Akt}$ signaling pathway is involved in the regulation of invasion and migration by STIM1 is unclear and needs to be further explored.

In the present study, we determined whether STIM1 knockdown inhibited cell migration and invasion of prostate cancer and further explored the potential underlying mechanism of STIM1 in the process with a focus on the regulation of the PI3K/Akt signaling pathway.

\section{Materials and methods}

Reagents. The PI3K inhibitor LY294002 was obtained from Selleckchem (Houston, TX, USA). Matrigel was purchased from BD Biosciences (San Jose, CA, USA), Transwell Minicells were obtained from Millipore (Darmstadt, Germany). A BCA protein qualification kit was purchased from Pierce (Rockford, IL, USA). The anti-STIM1 antibody for immunohistochemistry (IHC) staining was purchased from Abgent (San Diego, CA, USA). Anti-STIM1 (4916), anti-p-Akt (Thr308) (13038), anti-t-Akt (4691), anti-GAPDH (5174), anti-E-cadherin (3195), anti-N-cadherin (13116), anti-vimentin (5741), anti-Snail (3879) and secondary antibodies anti-rabbit (14708) and anti-mouse (14709) antibodies were obtained from Cell Signaling Technology (Boston, MA, USA).

Prostate tissue acquisition and IHC staining. Prostate cancer specimens were obtained from prostate cancer patients undergoing prostatectomy or prostate biopsy. Benign prostatic hyperplasia $(\mathrm{BPH})$ tissues were obtained from $\mathrm{BPH}$ patients undergoing surgery at the Second Affiliated Hospital of Soochow University and were used as a normal control. The present study was approved by the Research Ethics Committee of the Second Affiliated Hospital of Soochow University and was in compliance with the Helsinki Declaration. Tissues were examined by pathologists to confirm the diagnosis before IHC analyses. All specimens were fixed in $10 \%$ formalin, embedded in paraffin, and cut into $4-\mu \mathrm{m}$ thick slides. The slides were dewaxed, and the endogenous peroxidase activity was blocked by treatment with $3 \%$ hydrogen peroxide solution in methanol for $20 \mathrm{~min}$. Non-specific binding was prevented by blocking with normal goat serum (1:100) for $30 \mathrm{~min}$. The staining procedure was carried out using the avidin-biotin-peroxidase complex method. The expression of STIM1 was evaluated by staining with a mouse anti-STIM1 antibody. After incubation with a primary antibody for $60 \mathrm{~min}$, the slides were washed using phosphate-buffered saline (PBS) 3 times, and then incubated with a biotinylated goat anti-mouse $\operatorname{IgG}(\mathrm{H}+\mathrm{L})$ at $37^{\circ} \mathrm{C}$ for $30 \mathrm{~min}$, followed by incubation with a streptavidin-biotinylated HRP complex (Sigma, St. Louis, MO, USA) for $30 \mathrm{~min}$. Reactive products were visualized with 3,3'-diaminobenzidene (DAB) as the chromogen, and slides were counterstained with hematoxylin. Sections previously known to express STIM1 were included in each run, receiving either the primary antibody as the positive control or a mouse $\operatorname{IgG}$ as the negative control. Stained slides were observed by microscope.

All slides were evaluated twice at different time-points by two independent pathologists. The expression level of STIM1 was assigned a score based on the percentage of positive tumor cells over total tumor cells and their staining intensities. The proportion of positive tumor cells $\leq 10 \%, 11-25 \%, 26-50 \%$ and $>50 \%$ were scored as 1, 2, 3 and 4 . In addition, when the staining intensities were a non-significant brown, a slight brown, a moderate brown and a deep brown, they were scored as 1, 2, 3 and 4. Then, the two scores were added. A score of 2-3 was graded as weak, and a score of 4-8 was graded as strong.

Cell culture. Normal prostate cell line RWPE-1 and prostate cancer cell lines LNCap, PC-3 and DU-145 were purchased from the American Type Culture Collection (ATCC; Manassas, VA, USA). These were cultured according to the instructions of ATCC. C4-2 was obtained from UroCor Inc., (Oklahoma City, OK, USA) and grown in RPMI-1640 medium containing $10 \%$ fetal bovine serum (FBS) (Atlanta Biologicals, Flowery Branch, GA, USA) and 1\% penicillin-streptomycin solution (Invitrogen, Carlsbad, CA, USA). The cells were observed and images were obtained using an inverted microscope (Olympus, Tokyo, Japan). When the cell density reached $90-100 \%$, the cells were sub-cultured and seeded to plates according to the experimental design. The culture medium was replaced by fresh one every 2-3 days or according to the experimental design.

Total RNA isolation, cDNA reversion and polymerase chain reaction $(P C R)$. Total RNA was isolated using Trizol reagent (Invitrogen) according to the manufacturer's instructions. The concentration of total RNA was detected by UV spectrophotometry. RT-PCR was performed by the two-step method. Synthesis of cDNA was performed using a cDNA Synthesis kit (Thermo Fisher Scientific, Franklin Lakes, NJ, USA). The PCR reaction conditions were: $95^{\circ} \mathrm{C}$ for $5 \mathrm{~min}, 94^{\circ} \mathrm{C}$ for $30 \mathrm{sec}$, $56^{\circ} \mathrm{C}$ for $30 \mathrm{sec}, 72^{\circ} \mathrm{C}$ for $30 \mathrm{sec}$ for 40 cycles; the total volume was $20 \mu \mathrm{l}$. GAPDH was used as an internal standard. The sequences of the primers used were: GAPDH forward, TGTGG GCATCAATGGATTTGG and reverse, ACACCATGTATTC CGGGTCAAT; and STIM1 forward, AGTCACAGTGAGAA GGCG AC and reverse, ACACCATGTATTCCGGGTCAAT. All experiments were performed in triplicate.

Western blot analysis. Total protein was extracted by radio immunoprecipitation assay (RIPA) buffer $(0.15 \mathrm{mM} \mathrm{NaCl}$, $0.05 \mathrm{mM}$ Tris- $\mathrm{HCl}, \mathrm{pH} 7.5,1 \%$ Triton, $0.1 \%$ SDS, $0.1 \%$ sodium deoxycholate and $1 \%$ NP40). Sample extracts (30 $\mu \mathrm{g})$ were loaded to $12 \%$ SDS-polyacrylamide gels (PAGE) using a minigel apparatus and transferred to polyvinylidene difluoride (PVDF) membranes (both from Bio-Rad, Hercules, CA, USA). The membranes were blocked for $1 \mathrm{~h}$ with $5 \%$ skim milk, then incubated overnight with primary antibody. Then the membranes were washed using Tris-buffered saline with Tween-20 (TBST) 3 times and incubated with a secondary antibody for $1 \mathrm{~h}$ at room temperature. Blots were visualized by enhanced chemiluminescence (ECL) system.

Construction and infection of lentivirus-mediated short hairpin vector. For short hairpin RNA (shRNA)-mediated knockdown of STIM1, cells were transfected with lentiviral particles produced using the STIM1-pGCSIL-GFP plasmid 
A
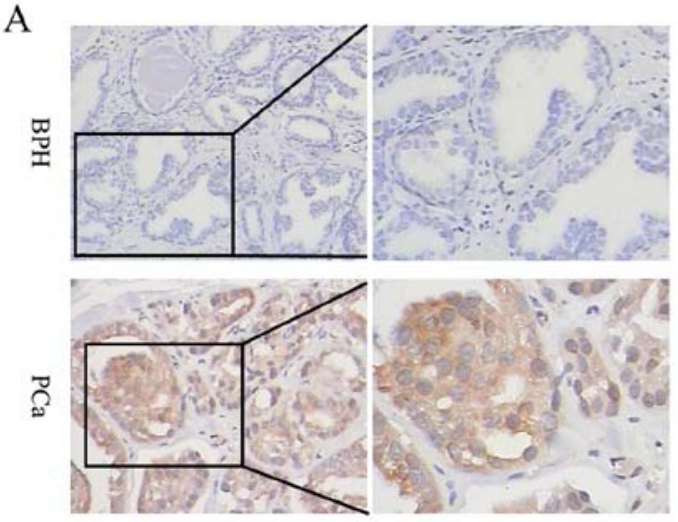

D

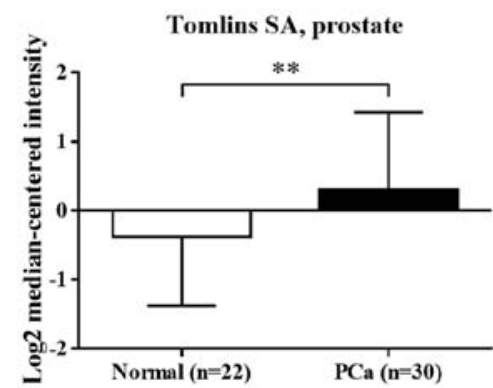

B

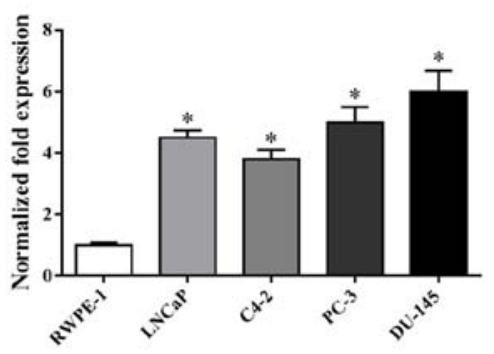

C
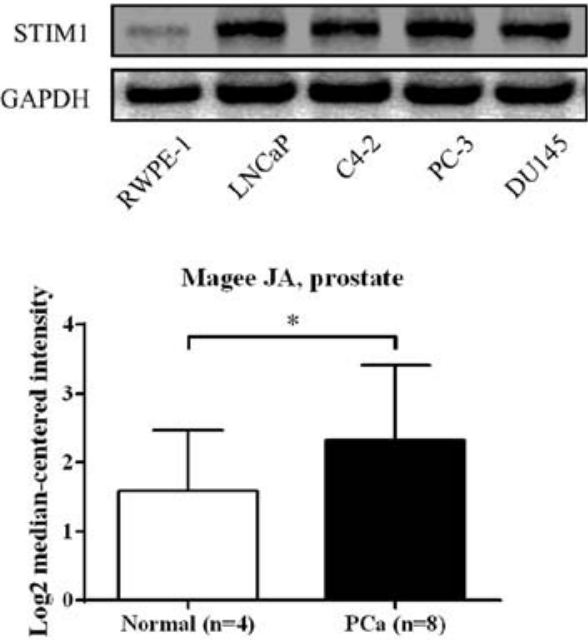

Figure 1. STIM1 is upregulated in PCa. (A) The expression of STIM1 in BPH tissues and prostate cancer tissues was detected by immunohistochemistry. High expression levels of STIM1 were observed in prostate cancer tissues. The expression of STIM1 was low in BPH tissues. (B and C) The expression of STIM1 in normal prostate cell line RWPE-1 and prostate cancer cell lines LNCap, C4-2, PC-3 and DU-145 were determined by (B) RT-PCR at the mRNA level and (C) western blotting at the protein level. GAPDH was used as an internal control. (D) The expression of STIM1 in samples from the Oncomine database; ${ }^{*} \mathrm{P}<0.05,{ }^{* *} \mathrm{P}<0.01$. STIM1, stromal-interacting molecule 1; PCA, prostate cancer; BPH, benign prostatic hyperplasia.

purchased from GeneChem Corporation (Shanghai, China). The targeting senses of the shRNAs were: shSTIM1-1, 5'-GCT CTCCACATTTGGATTCTT-3'; and shSTIM1-2, 5'-GGAGG ATAATGGCTCTATT-3'. The negative control was a double-stranded shRNA without sequence homology to any known human genes. For gene silencing, purified lentiviruses (shSTIM1-1 and shSTIM1-2) were added to cells at a multiplicity of infection of 20 for $8 \mathrm{~h}$, and was washed twice with medium. Infection with a multiplicity of infection of 20 resulted in a $>90 \%$ infection of cancer cells after $72 \mathrm{~h}$, as monitored by GFP expression. Therefore, we used a multiplicity of infection of 20 for the lentivirus in all of the experiments, as it yielded optimal knockdown of the gene in the required time. Control cells were infected with a negative control shRNA, as a vector control according to the same protocol.

Wound healing assay. Cells were seeded to 6-well plates at a concentration of $5 \times 10^{5}$ cells/well and incubated overnight. The cells were then infected with lentiviruses and incubated for an additional $48 \mathrm{~h}$ until the cell monolayers formed. The cells were starved by serum-free medium overnight. The wounds were scratched by sterile $200 \mu$ l pipet tips. After scratching, the cells were washed with PBS twice and cultured in serum-free medium. Images of the wounds were captured at time-points of 0,12 and $24 \mathrm{~h}$ by an inverted microscope (magnification, $\mathrm{x} 40$ ).

Transwell migration and invasion assays. A Transwell migration assay was performed using Transwell chambers consisting of $8 \mu \mathrm{m}$ membrane filter inserts (Corning, Corning NY, USA). Matrigel was diluted in serum-free medium (1:5) and pre-paved to insert membranes $4 \mathrm{~h}$ before cell seeding. The cells were infected with a lentivirus for $72 \mathrm{~h}$, and then re-suspended in serum-free medium. The cells in $500 \mu 1$ serum-free medium ( $3 \times 10^{4}$ cells for migration and $10 \times 10^{4}$ cells for invasion) were added to the upper chamber, and the lower chamber was filled with $1 \mathrm{ml}$ normal culture medium containing 10\% FBS. After incubation for $24 \mathrm{~h}$, the cells were fixed with $4 \%$ paraformaldehyde and stained with crystal violet. Migrated and invaded cells were observed using a microscope (magnification, x100) and images were captured.

Statistical analysis. To carry out statistical analysis, we used the software SPSS 23 (SPSS, Inc., Chicago, IL, USA) and GraphPad Prism 6 (GraphPad Software, San Diego, CA, USA). The correlation between STIM1 expression and clinicopathological parameters was analyzed by Chi-square $\left(\chi^{2}\right)$ test. The rest of the experimental data were represented from at least 3 independent experiments. Statistical significance was evaluated using the Student's t-test. A P-value $<0.05$ was considered to indicate a statistically significant result.

\section{Results}

STIM1 is overexpressed in prostate cancer tissues. To evaluate the expression of STIM1 in human prostate cancer tissues and benign prostate tissues (BPH), we examined the specimens by IHC. As shown in Fig. 1A, the expression of STIM1 in prostate 
A

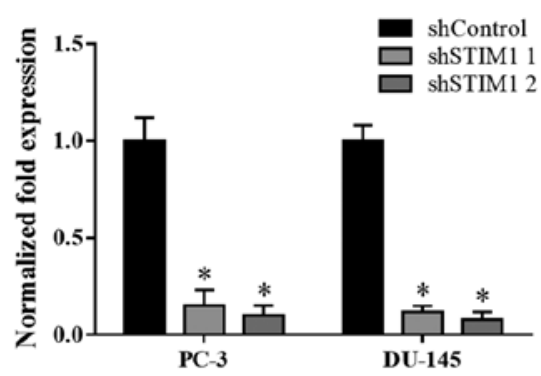

B

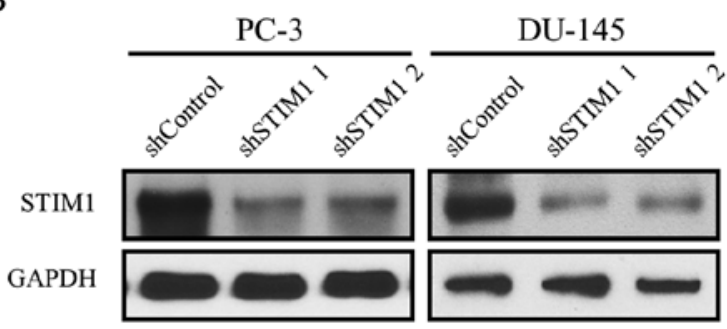

$\mathrm{C}$

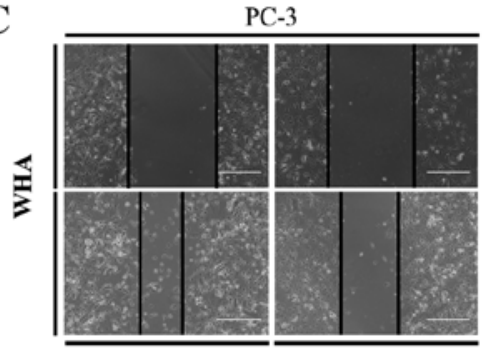

shControl

$\mathrm{D}$

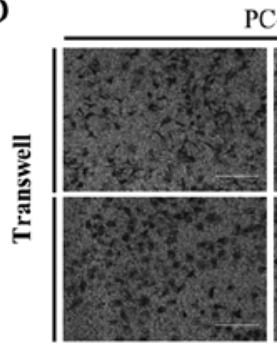

shControl

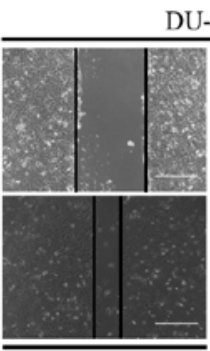

shControl

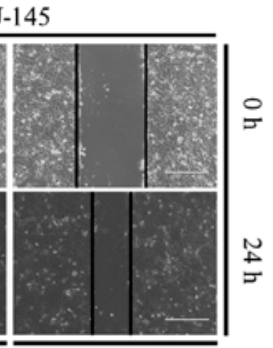

shSTIMl

DU-145

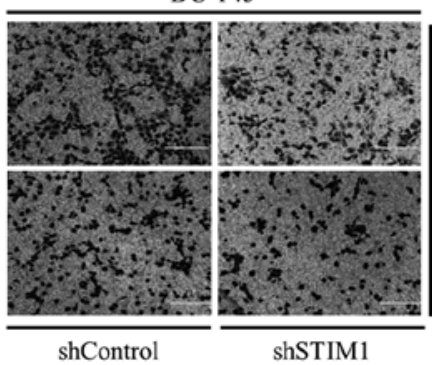

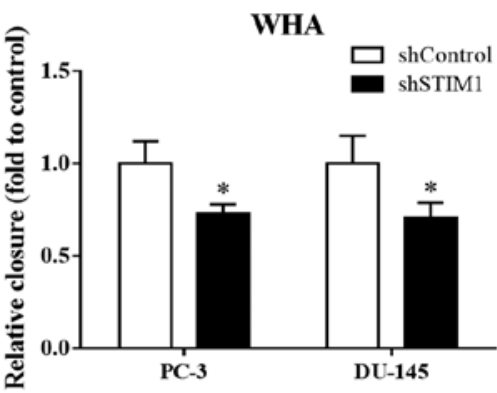

Transwell

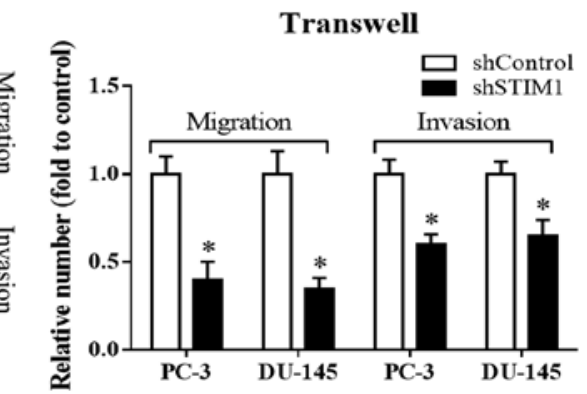

Figure 2. STIM1 knockdown inhibits cell migration and invasion. (A and B) Two independent STIM1 shRNAs and shControl were transfected into PC-3 and DU-145 cells. The knockdown efficiency was determined by (A) RT-PCR and (B) western blotting. (C and D) A wound healing assay and a Transwell assay were performed to study the influence of STIM1 knockdown on cell migration and invasion. The statistical analyses are shown in C and D; ${ }^{\mathrm{P}}<0.05$. STIM1, stromal-interacting molecule 1.

cancer was higher than benign prostate hyperplasia $(\mathrm{BPH})$ tissues. Overall, STIM1 was expressed in 3 out of $10(30.0 \%)$ BPH specimens; whereas it was expressed in 37 out of 47 prostate cancer specimens (78.7\%). The difference was statistically significant $(\mathrm{P}=0.007)$. We also searched in databases such as Oncomine (www.oncomine.org) and found studies conducted by Magee et al (22) and Tomlin et al (23) which revealed similar results (Fig. 1D).

To study whether STIM1 was correlated to prostate cancer progression, the association between STIM1 expression and clinicopathological characteristics was analyzed. As shown in Table I, the expression of STIM1 was found to be significantly associated with stage grouping. The STIM1 expression level of stage I+II was significantly lower than that of stage III+IV $(\mathrm{P}=0.016)$. However, there was no significant correlation between STIM1 expression and age or prostate cancer Gleason score $(\mathrm{P}>0.05)$.

We also investigated the expression of STIM1 in cell lines derived from normal prostate or prostate cancers. As shown in Fig. 1B and C, the expression of STIM1 in prostate cancer cell lines was much higher than that in normal prostate epithelial cell line (RWPE-1) at both the mRNA and protein levels. These results indicate that STIM1 may be an oncogene in prostate tumorigenesis.
Table I. Association between STIM1 expression and clinicopathological features in patients recruited.

\begin{tabular}{lcccc}
\hline & \multicolumn{3}{c}{$\begin{array}{c}\text { STIM1 } \\
\text { expression }\end{array}$} \\
\cline { 3 - 4 } Characteristics & No. & Weak & Strong & P-values \\
\hline Diagnosis & & & & 0.007 \\
$\quad$ BPH & 10 & 7 & 3 & \\
PCa & 47 & 10 & 37 & \\
Age (PCa) & & & & 0.487 \\
$\quad$ 65 & 19 & 5 & 14 & \\
$>65$ & 28 & 5 & 23 & \\
PCa Gleason score & & & & 0.709 \\
$\quad$ 7 & 14 & 2 & 12 & \\
$>7$ & 33 & 8 & 25 & 0.016 \\
PCa stage grouping & & & & \\
I+II & 12 & 6 & 6 & \\
III+IV & 35 & 4 & 31 & \\
\hline
\end{tabular}

STIM1, stromal-interacting molecule 1; $\mathrm{BPH}$, benign prostatic hyperplasia; $\mathrm{PCa}$, prostate cancer. 


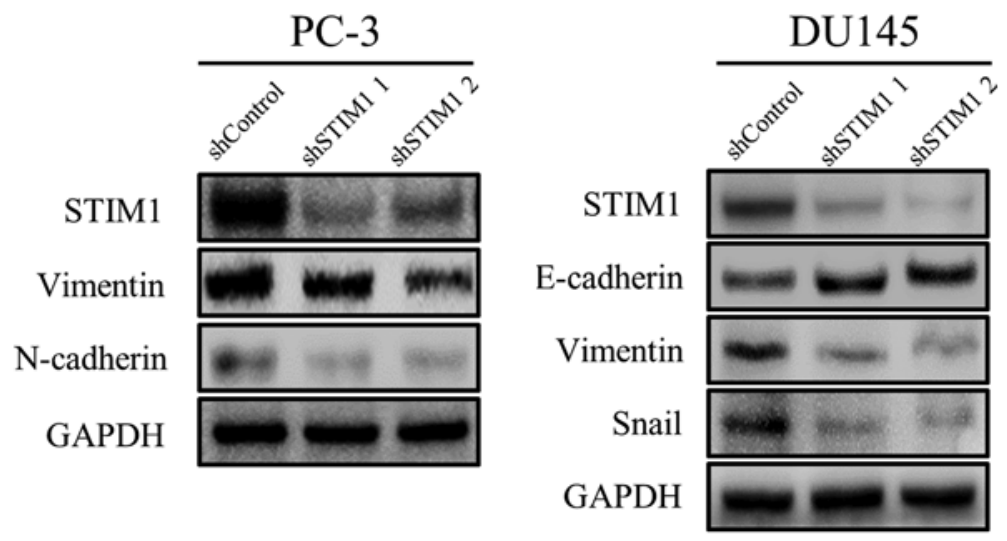

Figure 3. Motility inhibition by STIM1 knockdown is associated with EMT suppression. STIM1 was knocked down by two independent shRNAs in PC-3 and DU-145 cells. EMT markers E-cadherin, N-cadherin, vimentin and Snail were assessed by western blotting. STIM1, stromal-interacting molecule 1.

A

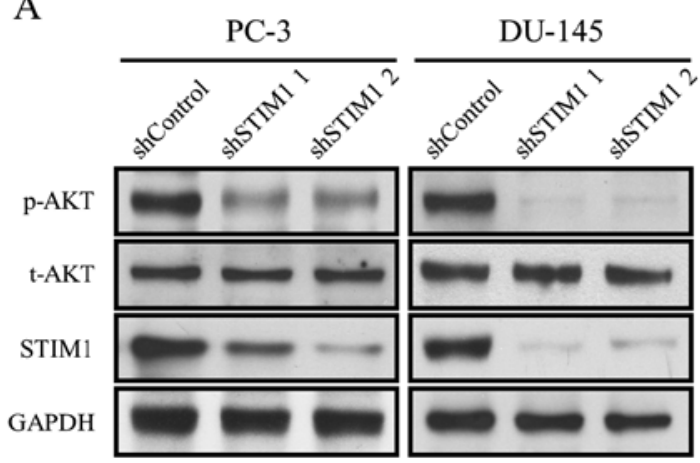

B

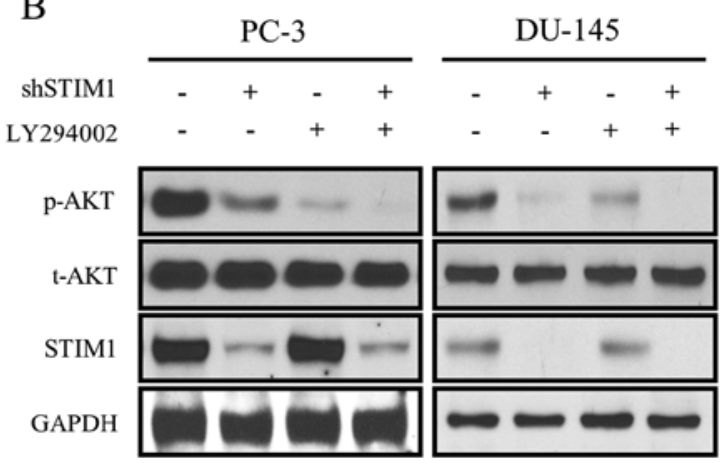

Figure 4. STIM1 knockdown inactivates PI3K-Akt. (A) Two independent shSTIM1s and shControl were transfected into PC-3 and DU-145 cells for 3 days and whole cell extracts were isolated. The expression of p-Akt, t-Akt and STIM1 was determined by western blotting. (B) shSTIM1 and shControl were transfected into PC-3 and DU-145 cells for 2 days, and then the culture medium was replaced with fresh medium with/without $10 \mu \mathrm{M}$ LY294002. Protein was harvested after $24 \mathrm{~h}$ of treatment and the expression of p-Akt, t-Akt and STIM1 was detected by western blotting. GAPDH was used as a loading control. STIM1, stromal-interacting molecule 1.

STIM1 knockdown inhibits migration and invasion in prostate cancer cells. To study the role of STIM1 in prostate cancer, we first designed two independent STIM1 shRNAs to knock down STIM1 in PC-3 and DU-145 cell lines. A double-stranded shRNA without sequence homology to any known human genes was used as control (Fig. 2A and B).

Wound healing assay and Transwell migration were then utilized to study the impact of STIM1 knockdown on cell migration. As shown in Fig. 2C and D, the STIM1 knockdown groups exhibited less wound closure and migrated cells as compared to the shControl, indicating that cell migration was inhibited when STIM1 was knocked down. Cell invasion was also suppressed by STIM1 knockdown as determined by Transwell invasion assay (Fig. 2D).

Motility inhibition by STIMI knockdown is associated with EMT suppression. To understand the potential mechanism involved in the suppressed migration and invasion by STIM1 knockdown, we examined EMT-related markers since STIM1 has been reported to promote cancer cell metastasis through the induction of EMT. As shown in Fig. 3, vimentin and $\mathrm{N}$-cadherin were downregulated by STIM1 knockdown in PC-3 cells. In DU-145 cells, STIM1 knockdown decreased the expression of vimentin and Snail but increased the expression of E-cadherin. These results demonstrated that the migration and invasion inhibited by STIM1 knockdown were associated with EMT suppression.

The PI3K/Akt signaling pathway is inactivated by STIMI knockdown. To study the influence of STIM1 knockdown on the PI3K/Akt signaling pathway, we assessed the expression of p-Akt, t-Akt and STIM1 in PC-3 and DU-145 cells after $72 \mathrm{~h}$ of infection with shSTIM1. As shown in Fig. 4A, p-Akt (Thr308) was suppressed by STIM1 knockdown with no change observed in t-Akt. To further confirm the involvement of PI3K/Akt, LY294002, a classic PI3K inhibitor, was used. As shown in Fig. 4B, both LY294002 and STIM1 decreased the levels of p-Akt without affecting the expression of t-Akt. When these two reagents were used in combination, the PI3K/Akt inhibition effect was further enhanced (Fig. 4B).

These results revealed that the PI3K/Akt signaling pathway was suppressed by STIM1 knockdown in prostate cancer cells.

The PI3K/Akt signaling pathway is involved in the inhibition of migration and invasion induced by STIM1 knockdown. To determine whether PI3K/Akt inactivation mediated the suppression of STIM1 knockdown on cell migration and invasion, we detected cell migration under the treatment of 


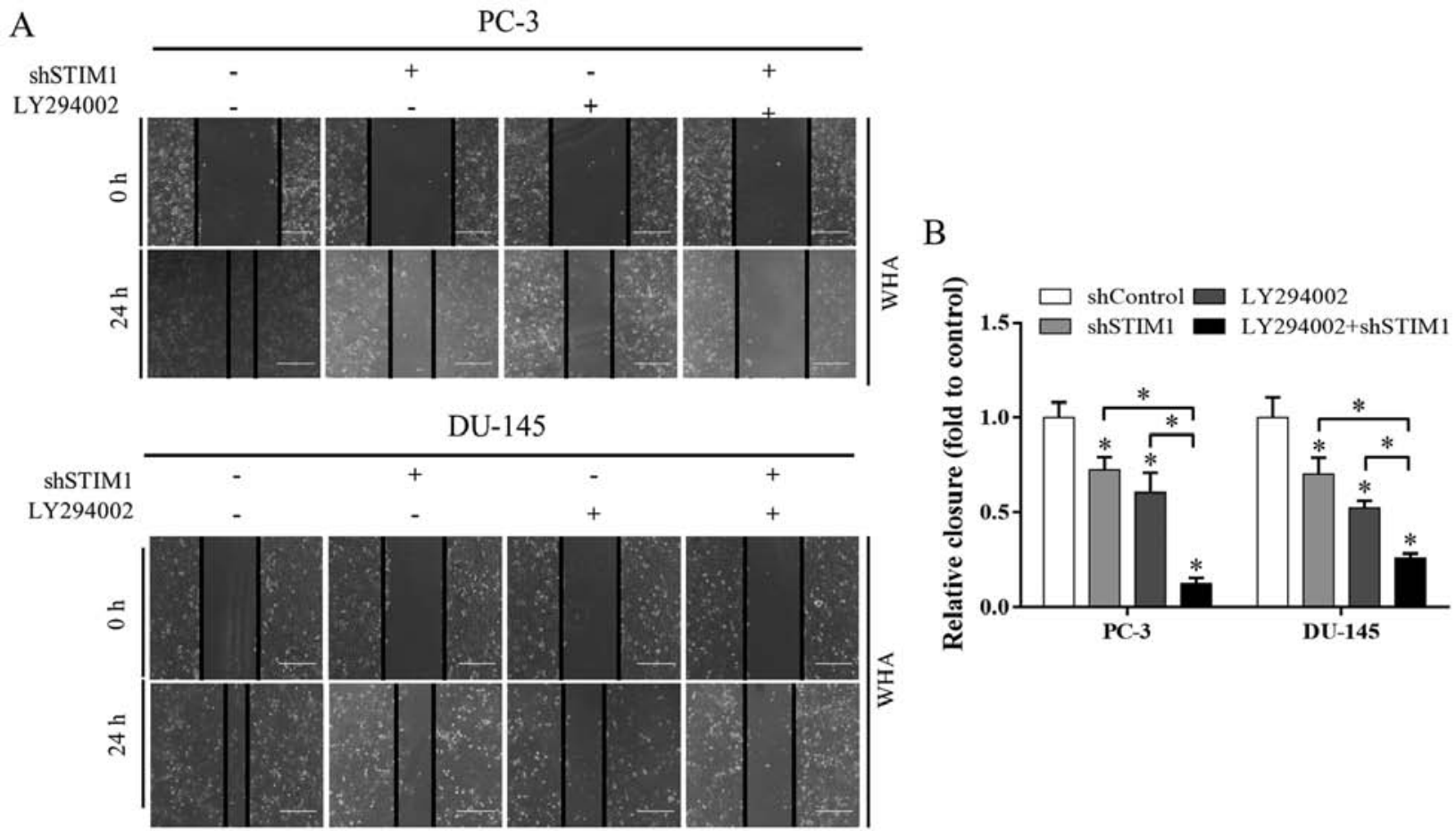

Figure 5. The PI3K/Akt signaling pathway is involved in the inhibition of migration and invasion induced by STIM1 knockdown. PC-3 and DU-145 cells were transfected with shSTIM1 and shControl for $48 \mathrm{~h}$, and then treated with culture medium with/without $10 \mu \mathrm{M} \mathrm{LY} 294002$ for an additional $24 \mathrm{~h}$. (A and B) Cell migration was determined by wound healing assay; ${ }^{*} \mathrm{P}<0.05$. STIM1, stromal-interacting molecule 1.

A

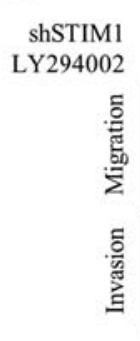

B

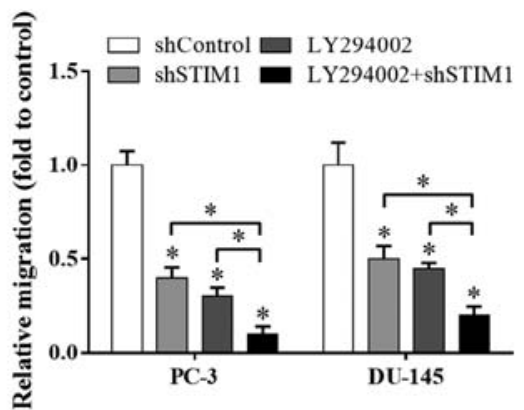

DU-145

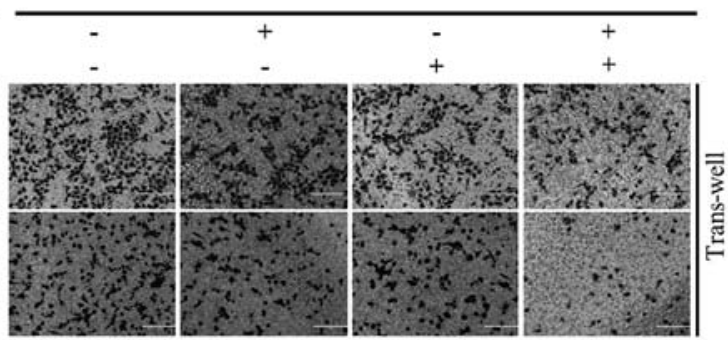

$\mathrm{C}$

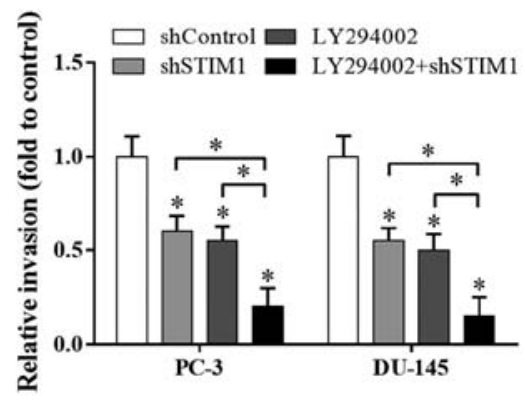

Figure 6. The PI3K/Akt signaling pathway is involved in the inhibition of migration and invasion induced by STIM1 knockdown. PC-3 and DU-145 cells were transfected with shSTIM1 and shControl for $48 \mathrm{~h}$, and then treated with culture medium with/without $10 \mu \mathrm{M} \mathrm{LY} 294002$ for an additional $24 \mathrm{~h}$. (A and B) Cell migration was determined by Transwell migration assay. (A and C) Cell invasion was determined by Transwell invasion assay; "P<0.05. STIM1, stromalinteracting molecule 1 .

LY294002 and/or STIM1 knockdown. Both LY294002 and STIM1 inhibited cell migration, and when these two treatments were used in combination, cell migration inhibition was markedly enhanced, as determined by wound healing assay (Fig. 5) and Transwell migration assay (Fig. 6A and B).
A similar phenomenon was observed in the Transwell invasion assay when studying cell invasion (Fig. 6A and C).

These data reveal that inactivation of PI3K/Akt is a potential underlying mechanism involved in migration and invasion inhibition induced by STIM1 knockdown. 


\section{Discussion}

Calcium signaling regulates a variety of cellular functions by activating or inhibiting cellular genes and signaling pathways $(24,25)$. A number of studies have documented that tumor progression is generally associated with dysregulated expression of $\mathrm{Ca}^{2+}$ channels and other molecules involved in $\mathrm{Ca}^{2+}$ homeostasis (26-28). STIM1 is one of the important components of major $\mathrm{Ca}^{2+}$ entry in non-excitable cells and has been reported to be aberrantly expressed in various types of cancer. STIM1 plays an important role in cell proliferation, migration and invasion in cervical cancer, hepatocarcinoma, glioblastoma and gastric cancer. Thus, STIM1 is considered to be an oncogene and a potential therapeutic target for these types of cancer $(29,30)$. A recent study revealed that STIM1 was downregulated in prostate cancer (31), which was contrary to other studies and databases. Thus, there is a need to clarify the exact role of STIM1 in prostate cancer and further explore its functions. In the present study, we found that STIM1 was upregulated both in prostate cancer tissues and prostate cancer cell lines. STIM1 played a pivotal role in prostate cancer cell migration and invasion as STIM1 knockdown decreased both cell migration and invasion. We then examined EMT-related markers and found that mobility inhibited by STIM1 knockdown was associated with EMT reversion.

Increasing studies indicate that $\mathrm{Ca}^{2+}$ is an important regulator of the PI3K/Akt signaling pathway, and an increase of intracellular $\mathrm{Ca}^{2+}$ activates the PI3K/Akt pathway (32-37). PI3K/Akt signaling is one of the most important intracellular pathways regulating cellular homeostasis. PI3K/Akt is over-activated in a various types of cancer, and is associated with progression of malignancy and, in particular, metastasis. Inhibition of the PI3K/Akt pathway was revealed to suppress invasion and migration in a variety of cancer models and was considered to be a potential therapeutic approach to combat cancer metastases (38-40).

However, in prostate cancer, whether migration and invasion which were regulated by STIM1 involved the PI3K/Akt pathway is still uncertain. Our mechanistic study revealed that the PI3K/Akt signaling pathway was inactivated by STIM1 knockdown and was involved in the inhibition of migration and invasion induced by STIM1. In addition, the effect of STIM1 depletion was further enhanced by the combination with LY294002, suggesting that STIM1 knockdown inhibits the migration and invasion of prostate cancer cells involving the PI3K/Akt signaling pathway. However, besides the PI3K/Akt pathway, some other signaling pathways are also sensitive to calcium level changes within cells, and have been reported to be regulated by STIM1, such as the MAPK and AMPK pathways. Knockdown of STIM1 attenuates cytosolic calcium and subsequently increases AMPK phosphorylation (41). Furthermore, AMPK is a regulator of STIM1. STIM1 phosphorylation was reported to be mediated by the AMPK-p38 MAPK signaling axis (42). These signaling pathways differ from each other, but also have cross-talks. They work together to form a complex network while STIM1 is an important trigger. Our study just elucidated a part of the picture and more light is needed to be shed on the detailed mechanism involved in the future.

Collectively, our results indicated that STIM1 knockdown inhibited the migration and invasion of prostate cancer cells involving the inactivation of PI3K/Akt signaling pathway. These findings shed new light on our understanding of STIM1 and suggest that STIM1 may be a potential target in the prevention of prostate cancer metastasis.

\section{Acknowledgements}

The present study was supported by the Foundation of Health Bureau of Suzhou (SYSD2012084), the Natural Science Foundation of Jiangsu Province (BK20161222) and the Suzhou Foundation for the Development of Science and Technology (SYS201629), P.R. China.

\section{References}

1. Siegel RL, Miller KD and Jemal A: Cancer statistics, 2016. CA Cancer J Clin 66: 7-30, 2016.

2. Lucas JM, Heinlein C, Kim T, Hernandez SA, Malik MS, True LD, Morrissey C, Corey E, Montgomery B, Mostaghel E, et al: The androgen-regulated protease TMPRSS2 activates a proteolytic cascade involving components of the tumor microenvironment and promotes prostate cancer metastasis. Cancer Discov 4: 1310-1325, 2014.

3. Shah ET, Upadhyaya A, Philp LK, Tang T, Skalamera D, Gunter J, Nelson CC, Williams ED and Hollier BG: Repositioning 'old' drugs for new causes: Identifying new inhibitors of prostate cancer cell migration and invasion. Clin Exp Metastasis 33: 385-399, 2016.

4. Abdullaev IF, Bisaillon JM, Potier M, Gonzalez JC, Motiani RK and Trebak M: Stim1 and Orai1 mediate CRAC currents and store-operated calcium entry important for endothelial cell proliferation. Circ Res 103: 1289-1299, 2008.

5. Berridge MJ, Lipp P and Bootman MD: The versatility and universality of calcium signalling. Nat Rev Mol Cell Biol 1: 11-21, 2000.

6. Hu J, Qin K, Zhang Y, Gong J, Li N, Lv D, Xiang R and Tan X: Downregulation of transcription factor Oct4 induces an epithelial-to-mesenchymal transition via enhancement of $\mathrm{Ca}^{2+}$ influx in breast cancer cells. Biochem Biophys Res Commun 411: 786-791, 2011.

7. Lee KP, Yuan JP, Hong JH, So I, Worley PF and Muallem S: An endoplasmic reticulum/plasma membrane junction: STIM1/ Orail/TRPCs. FEBS Lett 584: 2022-2027, 2010.

8. Yang S, Zhang JJ and Huang XY: Orail and STIM1 are critical for breast tumor cell migration and metastasis. Cancer Cell 15: 124-134, 2009.

9. Chen YF, Chiu WT, Chen YT, Lin PY, Huang HJ, Chou CY, Chang HC, Tang MJ and Shen MR: Calcium store sensor stromalinteraction molecule 1-dependent signaling plays an important role in cervical cancer growth, migration, and angiogenesis. Proc Natl Acad Sci USA 108: 15225-15230, 2011.

10. Yang N, Tang Y, Wang F, Zhang H, Xu D, Shen Y, Sun S and Yang G: Blockade of store-operated $\mathrm{Ca}^{2+}$ entry inhibits hepatocarcinoma cell migration and invasion by regulating focal adhesion turnover. Cancer Lett 330: 163-169, 2013.

11. Li G, Zhang Z, Wang R, Ma W, Yang Y, Wei J and Wei Y: Suppression of STIM1 inhibits human glioblastoma cell proliferation and induces G0/G1 phase arrest. J Exp Clin Cancer Res 32: 20, 2013

12. King D, Yeomanson D and Bryant HE: PI3King the lock: Targeting the PI3K/Akt/mTOR pathway as a novel therapeutic strategy in neuroblastoma. J Pediatr Hematol Oncol 37: 245-251, 2015.

13. Chen X, Wang YW, Xing AY, Xiang S, Shi DB, Liu L, Li YX and Gao P: Suppression of SPIN1-mediated PI3K-Akt pathway by miR-489 increases chemosensitivity in breast cancer. J Pathol 239: 459-472, 2016.

14. Vander Broek R, Mohan S, Eytan DF, Chen Z and Van Waes C: The PI3K/Akt/mTOR axis in head and neck cancer: Functions, aberrations, cross-talk, and therapies. Oral Dis 21: 815-825, 2015.

15. Kim D, Kim S, Koh H, Yoon SO, Chung AS, Cho KS and Chung J: Akt/PKB promotes cancer cell invasion via increased motility and metalloproteinase production. FASEB J 15: 1953-1962, 2001. 
16. Yoeli-Lerner M, Yiu GK, Rabinovitz I, Erhardt P, Jauliac S and Toker A: Akt blocks breast cancer cell motility and invasion through the transcription factor NFAT. Mol Cell 20: 539-550, 2005.

17. Tanno S, Tanno S, Mitsuuchi Y, Altomare DA, Xiao GH and Testa JR: AKT activation up-regulates insulin-like growth factor I receptor expression and promotes invasiveness of human pancreatic cancer cells. Cancer Res 61: 589-593, 2001.

18. Mundi PS, Sachdev J, McCourt C and Kalinsky K: AKT in cancer: New molecular insights and advances in drug development. Br J Clin Pharmacol 82: 943-956, 2016.

19. Yang SX, Polley E and Lipkowitz S: New insights on PI3K/AKT pathway alterations and clinical outcomes in breast cancer. Cancer Treat Rev 45: 87-96, 2016.

20. Chen H, Zhou L, Wu X, Li R, Wen J, Sha J and Wen X: The $\mathrm{PI} 3 \mathrm{~K} / \mathrm{AKT}$ pathway in the pathogenesis of prostate cancer. Front Biosci 21: 1084-1091, 2016.

21. Foster K, Wang Y, Zhou D and Wright C: Dependence on PI3K/ Akt signaling for malignant rhabdoid tumor cell survival. Cancer Chemother Pharmacol 63: 783-791, 2009.

22. Magee JA, Araki T, Patil S, Ehrig T, True L, Humphrey PA, Catalona WJ, Watson MA and Milbrandt J: Expression profiling reveals hepsin overexpression in prostate cancer. Cancer Res 61 5692-5696, 2001.

23. Tomlins SA, Mehra R, Rhodes DR, Cao X, Wang L, Dhanasekaran SM, Kalyana-Sundaram S, Wei JT, Rubin MA, Pienta KJ, et al: Integrative molecular concept modeling of prostate cancer progression. Nat Genet 39: 41-51, 2007.

24. Wiegert JS and Bading H: Activity-dependent calcium signaling and ERK-MAP kinases in neurons: A link to structural plasticity of the nucleus and gene transcription regulation. Cell Calcium 49: 296-305, 2011

25. Müller I,Lipp P and Thiel G: $\mathrm{Ca}^{2+}$ signaling and gene transcription in glucose-stimulated insulinoma cells. Cell Calcium 52: 137-151, 2012.

26. Monteith GR, McAndrew D, Faddy HM and Roberts-Thomson SJ: Calcium and cancer: Targeting $\mathrm{Ca}^{2+}$ transport. Nat Rev Cancer 7 519-530, 2007.

27. Zhu M, Chen L, Zhao P, Zhou H, Zhang C, Yu S, Lin Y and Yang X: Store-operated $\mathrm{Ca}^{2+}$ entry regulates glioma cell migration and invasion via modulation of Pyk2 phosphorylation. J Exp Clin Cancer Res 33: 98, 2014.

28. Umemura M, Baljinnyam E, Feske S, De Lorenzo MS, Xie LH, Feng X, Oda K, Makino A, Fujita T, Yokoyama U, et al: Storeoperated $\mathrm{Ca}^{2+}$ entry (SOCE) regulates melanoma proliferation and cell migration. PLoS One 9: e89292, 2014

29. Wu Z, Qing J, Xia Y, Wang K and Zhang F: Suppression of stromal interaction molecule 1 inhibits SMMC7721 hepatocellular carcinoma cell proliferation by inducing cell cycle arrest. Biotechnol Appl Biochem 62: 107-111, 2015.

30. Xia J, Wang H, Huang H, Sun L, Dong S, Huang N, Shi M, Bin J, Liao Y and Liao W: Elevated Orail and STIM1 expressions upregulate MACC1 expression to promote tumor cell proliferation, metabolism, migration, and invasion in human gastric cancer. Cancer Lett 381: 31-40, 2016.
31. Xu Y, Zhang S, Niu H, Ye Y, Hu F, Chen S, Li X, Luo X, Jiang S, Liu Y, et al: STIM1 accelerates cell senescence in a remodeled microenvironment but enhances the epithelial-to-mesenchymal transition in prostate cancer. Sci Rep 5: 11754, 2015.

32. Zhang Y, Zhang T, Wu C, Xia Q and Xu D: ASICla mediates the drug resistance of human hepatocellular carcinoma via the $\mathrm{Ca}^{2+} / \mathrm{PI} 3-k i n a s e / A K T$ signaling pathway. Lab Invest 97: 53-69, 2017.

33. Li GW, Xing WJ, Bai SZ, Hao JH, Guo J, Li HZ, Li HX, Zhang WH, Yang BF, Wu LY, et al: The calcium-sensing receptor mediates hypoxia-induced proliferation of rat pulmonary artery smooth muscle cells through MEK1/ERK1,2 and PI3K pathways. Basic Clin Pharmacol Toxicol 108: 185-193, 2011.

34. Divolis G, Mavroeidi P, Mavrofrydi O and Papazafiri P: Differential effects of calcium on PI3K-Akt and HIF-1 $\alpha$ survival pathways. Cell Biol Toxicol 32: 437-449, 2016.

35. Liu ZM, Chen GG, Vlantis AC, Tse GM, Shum CK and van Hasselt CA: Calcium-mediated activation of PI3K and p53 leads to apoptosis in thyroid carcinoma cells. Cell Mol Life Sci 64: 1428-1436, 2007.

36. Wang C, Chi Y, Li J, Miao Y, Li S, Su W, Jia S, Chen Z, Du S, Zhang X, et al: FAM3A activates PI3K p110a/Akt signaling to ameliorate hepatic gluconeogenesis and lipogenesis. Hepatology 59: 1779-1790, 2014.

37. Danciu TE, Adam RM, Naruse K, Freeman MR and Hauschka PV: Calcium regulates the PI3K-Akt pathway in stretched osteoblasts. FEBS Lett 536: 193-197, 2003.

38. Ke L, Xiang Y, Guo X, Lu J, Xia W, Yu Y, Peng Y, Wang L, Wang G, Ye Y, et al: c-Src activation promotes nasopharyngeal carcinoma metastasis by inducing the epithelial-mesenchymal transition via PI3K/Akt signaling pathway: A new and promising target for NPC. Oncotarget 7: 28340-28355, 2016.

39. Niessner H, Schmitz J, Tabatabai G, Schmid AM, Calaminus C, Sinnberg T, Weide B, Eigentler TK, Garbe C, Schittek B, et al: PI3K pathway inhibition achieves potent antitumor activity in melanoma brain metastases in vitro and in vivo. Clin Cancer Res 22: 5818-5828, 2016.

40. Kim MS, Lee WS, Jeong J, Kim SJ and Jin W: Induction of metastatic potential by TrkB via activation of IL6/JAK2/STAT3 and PI3K/AKT signaling in breast cancer. Oncotarget 6: 40158-40171, 2015.

41. Mungai PT, Waypa GB, Jairaman A, Prakriya M, Dokic D, Ball MK and Schumacker PT: Hypoxia triggers AMPK activation through reactive oxygen species-mediated activation of calcium release-activated calcium channels. Mol Cell Biol 31: 3531-3545, 2011.

42. Sundivakkam PC, Natarajan V, Malik AB and Tiruppathi C: Store-operated $\mathrm{Ca}^{2+}$ entry (SOCE) induced by protease-activated receptor-1 mediates STIM1 protein phosphorylation to inhibit SOCE in endothelial cells through AMP-activated protein kinase and $\mathrm{p} 38 \beta$ mitogen-activated protein kinase. J Biol Chem 288: 17030-17041, 2013. 